\title{
Can simultaneous incentive contrast be produced by the pharmacological effects of alcohol?
}

\author{
W. MILES COX and JOHN E. MERTZ \\ University of Minnesota, Morris, Minnesota 56267
}

Three groups, each consisting of 10 female albino rats, were run in an experiment that tested for simultaneous incentive contrast effects by varying the alcohol content of fluids consumed prior to conditioning trials. All groups received five food pellets as reward in the runway. One control group drank an alcoholic fluid, and the second control group drank a nonalcoholic fluid. The experimental group drank the alcoholic fluid on some days and the nonalcoholic fluid on alternate days. Comparison of the appropriate experimental and control conditions revealed no positive contrast effect and only a marginal negative contrast effect in goal speeds.

Numerous studies conducted during the past 2 decades to test for simultaneous incentive contrast effects have found that a negative contrast effect regularly occurs, but a positive contrast effect is obtained only in special circumstances (Cox, 1975). One such set of circumstances was recently identified in our laboratory. In this study (Cox, 1981), alcoholic and nonalcoholic fluids that animals drank prior to their runway conditioning trials served as the sole cue for magnitude of food reward. Under these conditions, when experimental animals that were exposed to both large and small reward received the large reward in the presence of the alcoholic cue, a positive contrast effect occurred. When experimental animals received the small reward in the presence of the alcoholic cue, a negative contrast effect occurred.

Since in the Cox (1981) study alcohol both served as a cue and had pharmacological effects, the observed symmetrical incentive contrast could have resulted from either of these properties of alcohol, or a combination of them. In the present study, experimental animals again experienced a contrast between alcoholic and nonalcoholic fluids on alternate days, but unlike the earlier study, the fluid that was consumed did not predict the magnitude of food reward in the runway. Thus, here we determined whether simultaneous contrast could be produced by the pharmacological effects of alcohol apart from the cue properties that alcohol had in the earlier study.

\section{METHOD}

\section{Subjects}

The subjects were 30 female albino rats purchased from the Holtzman Company of Madison, Wisconsin, and were approximately 90 days old at the beginning of training.

\section{Apparatus}

The apparatus was a single straight runway that was painted flat gray and had clear Plexiglas ceilings. The interior was $10 \mathrm{~cm}$ in width and $119 \mathrm{~cm}$ in length, and the startbox, alley, and goalbox were $25.5 \mathrm{~cm}, 63 \mathrm{~cm}$, and $30.5 \mathrm{~cm}$ long, respectively. Guillotine doors separated the startbox and the goalbox from the alley. A food cup was placed at the end of the goalbox. Response latencies were measured with photoelectronic circuitry. Run and goal times were measured over a distance of $51 \mathrm{~cm}$ and $30 \mathrm{~cm}$, respectively.

\section{Procedure}

Upon receipt from the supplier, the animals were housed in individual home cages and were given ad-lib food and water until their weights had stabilized. Then the animals were placed on a food-deprivation regimen, consisting of $9 \mathrm{~g}$ of food each day, until their body weights were reduced to $85 \%$ of their ad-lib values. Thereafter, compensatory food was given whenever the weights fell below $85 \%$. Immediately prior to the experiment, the animals explored the runway for $5 \mathrm{~min}$ in groups of five animals each.

Three groups, consisting of 10 animals each, were run. One control group always drank an alcoholic fluid prior to the conditioning trials, and the other control group always drank a nonalcoholic fluid. The experimental group drank either the alcoholic or the nonalcoholic fluid according to a repetitive ANNA NAAN schedule for half of the animals and a repetitive NAAN ANNA schedule for the other half. All three groups received five $45-\mathrm{mg}$ Noyes food pellets on each conditioning trial. Each animal received four daily trials with an intertrial interval of about $15 \mathrm{~min}$. Training continued for 30 days.

As in our previous studies of the behavioral effect of alcohol (Cox, 1981; Cox \& Stainbrook, 1977), the animals were fluid deprived to facilitate consumption of the alcoholic fluid. Fluid deprivation began 5 days before experimental training. On the 1 st day, all animals were exposed to tap water for $30 \mathrm{~min}$. On the next 4 days, the animals had $15 \mathrm{~min}$ of exposure to the fluid that they would drink during training. The fluid was alcoholic for one control group, nonalcoholic for the other control group, and alcoholic or nonalcoholic for the experimental group, according to an ANNA schedule across days. The alcoholic fluid contained $4.8 \%$ alcohol, and both the alcoholic and nonalcoholic fluid contained $10 \%$ sucrose. The animals drank from graduated cylinders containing ball-bearing drinking spouts. During training, each squad of 10 animals drank the alcoholic or nonalcoholic fluid in their home cages for $15 \mathrm{~min}$ immediately prior to the daily runway trials. 


\section{RESULTS AND DISCUSSION}

Prior to analysis, latencies were converted into speeds $(1 / \mathrm{sec})$, and speeds for each animal's four daily trials were averaged. The data were then collapsed into 15 blocks of 2 days each. For the two control groups, each animal's speeds were averaged across the 2 days, thus yielding 10 scores/group for each block of 2 days. For the experimental group, the data from the five animals that were trained on each odd-numbered day under a given condition were grouped with the data from the five different animals that were trained on the following even-numbered day under that same condition, thus yielding 10 scores for each of the two experimental conditions for each block of 2 days.

Run and goal speeds yielded different information and are presented separately. Considering first the run speeds in Figure 1, we see negatively accelerated conditioning curves and asymptotic speeds that appear to have been reached well before the conclusion of training. It is further apparent from Figure 1 that the animals in the nonalcoholic control condition ran faster than animals in each of the other three conditions.

Since alcohol enhances the value of other rewards, the animals that consumed the food reward after having drunk the alcoholic fluid presumably had a more potent reward than animals that drank the nonalcoholic fluid. Comparing the two groups that therefore provide a test for positive contrast, alcoholic control and alcoholic experimental, we find that they did not differ $(F<1.0)$. On the other hand, the animals in the nonalcoholic experimental condition did run more slowly than the animals in the nonalcoholic control condition $[\mathrm{F}(1,18)$ $=4.70, \mathrm{p}<.04]$, a difference that is required for a negative contrast effect. However, in view of the facts that (1) the experimental animals did not run at different speeds when they drank the alcoholic and the nonalcoholic fluids and (2) the experimental animals ran at speeds comparable to those of the animals in the alcoholic control condition, the observed difference between the nonalcoholic/control and nonalcoholic/ experimental conditions cannot be interpreted as an incidence of negative contrast. In short, we have no basis

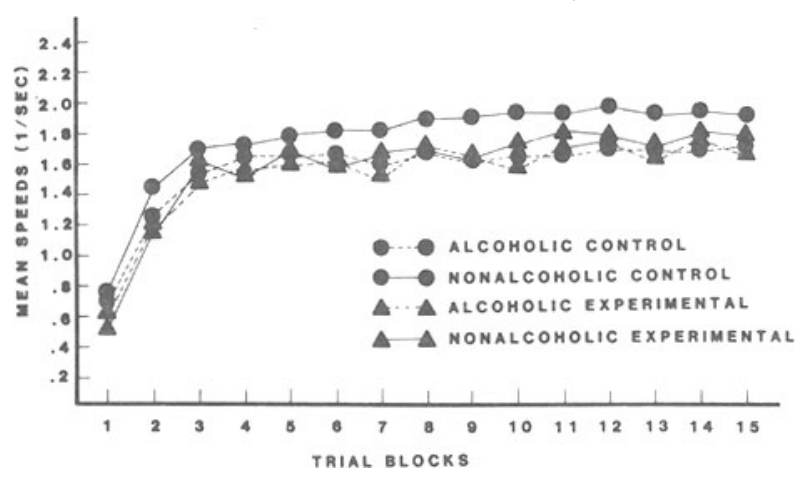

Figure 1. Mean run speeds of three groups (four conditions) as a function of trial blocks.

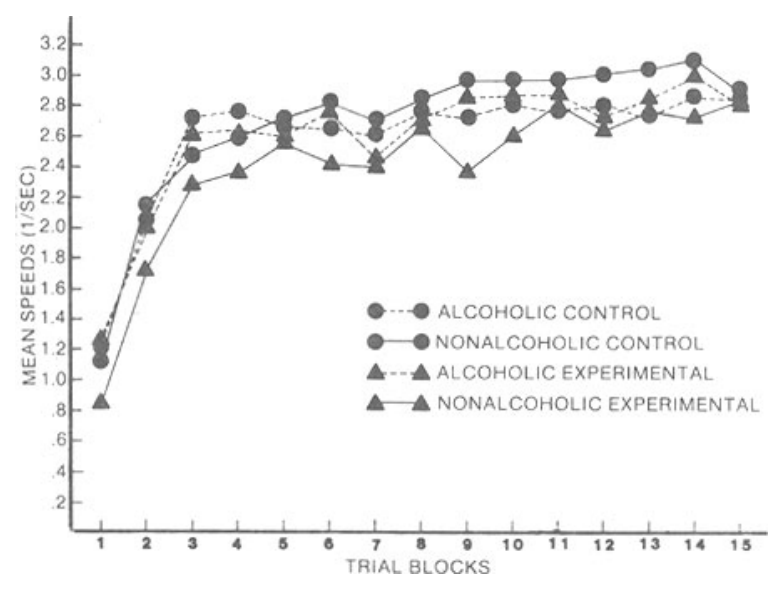

Figure 2. Mean goal speeds of three groups (four conditions) as a function of trial blocks.

for inferring that when the experimental animals drank the nonalcoholic solution they ran more slowly than the nonalcoholic control animals because they were contrasting the nonalcoholic condition with their more reinforcing alcoholic condition.

From the goal speeds in Figure 2, we see typical, negatively accelerated conditioning curves, with asymptotic speeds reached by the conclusion of training. As with run speeds, we see no indication here of a positive contrast effect. Animals trained under the alcoholic experimental condition did not run faster than animals trained under the alcoholic control condition $(\mathrm{F}<1.0)$. On the other hand, we do see a graphical representation of a negative contrast effect. Animals in the nonalcoholic experimental condition ran more slowly than animals in the nonalcoholic control condition during a large portion of training. Nevertheless, statistical analysis indicates that even during the middle portion of training, when graphically the negative contrast effect was most pronounced (i.e., on Trial Blocks 6-10), the nonalcoholic control and experimental groups differed only marginally $[\mathrm{F}(1,18)=3.40, \mathrm{p}<.08]$.

The different outcomes for run and goal speeds can perhaps be accounted for in terms of the differential influences of emotions on the animals' behavior at various points in the runway. That is, we would expect nonemotional effects to be more pronounced at points early in the runway and emotional effects to be more pronounced near the goal. Thus, what we may be observing in the run speeds is simple reductions in speeds by the two groups that drank the alcoholic fluid, resulting from the ataxic effects of alcohol. On the other hand, in the goal speeds, instead of response suppression due to alcohol, we see a marginal negative contrast effect that presumably results from the emotional consequences (e.g., frustration; Amsel, 1958, 1962) of animals' comparing their more and less favorable rewards.

Unlike the Cox (1981) study, in which symmetrical contrast effects occurred, in the present study we have no evidence whatsoever for positive contrast and only 
marginal evidence for negative contrast. Thus, from the present results, it appears that in the earlier study the cue rather than the pharmacological properties of alcohol was largely responsible for the observed symmetrical contrast effects. It is also possible that in studies of the effects of alcohol on runway behavior, the ataxic and emotional effects of alcohol tend to cancel each other, thus producing negative results such as the present ones.

\section{REFERENCES}

Amsel, A. The role of frustrative nonreward in noncontinuous reward situations. Psychological Bulletin, 1958, 55, 102-119.

Amsel, A. Frustrative nonreward in partial reinforcement and discrimination learning: Some recent history and a theoretical extension. Psychological Review, 1962, 69, 306-328.

Cox, W. M. A review of recent incentive contrast studies involving discrete-trial procedures. Psychological Record, 1975, 25, 373-393.

Cox, W. M. Simultaneous incentive contrast effects with alcoholic and nonalcoholic beverages as the discriminanda for reward magnitude. Physiological Psychology, 1981, 9, 276-280.

Cox, W. M., \& Stainbrook, G. L. Stress-induced alcohol consumption: A new paradigm. British Journal on Alcohol and Alcoholism, 1977, 12, 23-29.

(Received for publication October 29, 1982.) 